\title{
Sanguijuelas (Hirudinea: Glossiphoniidae) asociadas a Cryphiops caementarius (Decapoda: Palaemonidae) del Río Limarí, Chile
}

\author{
Patricio Tello, Viviane Jerez \& Viviana Olmos \\ Departamento de Zoología, Facultad de Ciencias Naturales y Oceanográficas, Universidad de Concepción, Casilla \\ 160-C, Concepción, Chile. Fax: 56-41-238982; ptello1305@latinmail.com; vijerez@udec.cl; volmos@udec.cl
}

Recibido 20-III-2003. Corregido 29-III-2006. Aceptado 25-X-2006.

\begin{abstract}
Leeches (Hirudinea: Glossiphoniidae) associated to Cryphiops caementarius (Decapoda: Palaemonidae) of the Limarí river, Chile. Two leech species were found parasitizing the shrimp Cryphiops caementarius from Limarí river, Chile. These ectoparasites can act as population regulators or development inhibitors in their host; however the biological characteristics of this host-parasite interaction are unknown. We analyze the inter-specific differences of the parasitism and its relation with host size and sex using quantitative descriptors. Abundance, prevalence, intensity and range were estimated with respect to host size and sex. The leeches belong to two species of Glossiphonidae: Helobdella triserialis and H. duplicata. Forty-seven percent of the hosts were parasitized by one or both leech species. H. triserialis was found only in the pleopod of the hosts with the highest prevalence values (42\%), intensity (2.9) and range (1-11). H. duplicata was found only in the branchial filaments with $7 \%$ prevalence, an intensity of 1.7 and a range of 1-4. The host's total length and sex can explain the variation in leech intensity and infection prevalence. However, when only the host's sex is considered, prevalence only increased with size in female hosts. Morphological characteristics of the females could explain the greater $H$. triserialis intensity in the base of the pleopod and would be related to a greater abdomen development. Rev. Biol. Trop. 55 (1): 255-260. Epub 2007 March. 31.
\end{abstract}

Key words: Helobdella, leeches, Palaemonidae, Cryphiops caementarius, ectoparasites, Chile.

El "camarón de río del norte", Cryphiops caementarius (Molina, 1782) es uno de los componentes biológicos más importantes de las aguas continentales de la vertiente occidental de América del Sur (Bahamonde y Vila 1971), cuya área de distribución se extiende entre los ríos Taymi-Mochami (Perú) $\left(6^{\circ} \mathrm{S}\right)$ y Maipo (Chile) (33 S) (Amaya y Guerra 1976, Jara 1997). Los tamaños poblacionales de esta especie están fuertemente influenciados por las precipitaciones, que hacen posible un importante reclutamiento proveniente de las zonas estuarinas o marinas. Sin embargo, en los últimos años se ha observado la disminución paulatina de estas poblaciones como consecuencia de la irregularidad del caudal de las aguas de los ríos que habitan y por las capturas a las que han sido sometidos (Bahamonde y Vila 1971). La principal estrategia de protección para estos organismos han sido las vedas sucesivas y totales, con resultados insuficientes en el intento de incrementar sus poblaciones naturales. Por esta razón, los servicios estatales encargados de recursos naturales renovables, han expresado su preocupación por conservar esta especie y estudiar la posibilidad de crear centros de crianza y repoblación (Meruane 1994).

Uno de los problemas más importantes en las estrategias de protección de especies, con fines económicos o de conservación, es predecir el posible efecto de parásitos sobre sus poblaciones (Calvo-Ugartebur y McQuaid 1998, Sasal et al. 2000). Los parásitos son capaces de castrar, y/o disminuir la fecundi- 
dad y en algunos casos, alterar la conducta de apareamiento de sus hospederos (Baudoin 1975, Muñoz y George-Nascimento 1999). Sin embargo y a pesar de la importancia de estos aspectos en planes de cultivo y explotación comercial, no existen reportes de parasitismo en C. caementarius. Estudios preliminares realizados en camarones provenientes del río Limarí, IV Región de Chile, permitieron detectar la presencia de dos especies de sanguijuelas del género Helobdella (Blanchard, 1849), parasitando adultos de $C$. caementarius. Usualmente, el impacto de los ectoparásitos sobre sus hospederos, es un resultado directo de su abundancia y alta tasa de reclutamiento (Coop et al. 2002); por ello y dado que estas sanguijuelas se alimentan de fluidos corporales y presentan cuidado parental de sus crías (Sawyer 1986, Gullo 2003), su presencia podría incorporar gastos adicionales al presupuesto energético del hospedero (Olmos y George-Nascimento 1997, Combes 2001), con consecuencias sobre los componentes de la adecuación biológica de C. caementarius. En este estudio se da a conocer las características básicas de este sistema hospedero-parásito y a través de descriptores cuantitativos del parasitismo, se determina si existen diferencias entre estas dos especies de sanguijuelas en relación con características del microhábitat y establecemos su relación con el tamaño corporal y sexo del hospedero.

\section{MATERIALES Y MÉTODOS}

Durante el año 2000, se recolectaron 165 ejemplares de $C$. caementarius en el río Limarí, entre las localidades de Ovalle $\left(30^{\circ} 37^{\prime} \mathrm{S}\right.$, $\left.71^{\circ} 15^{\prime} \mathrm{W}\right)$ y Limarí $\left(30^{\circ} 38^{\prime} \mathrm{S}, 71^{\circ} 12^{\prime} \mathrm{W}\right)$, Chile. Los individuos fueron individualizados y congelados a $-5{ }^{\circ} \mathrm{C}$ hasta el momento de ser examinados. En el laboratorio se registró la identidad específica de los camarones siguiendo a Retamal (1981), y luego todos fueron numerados, sexados y medida su longitud total (LT, de rostro a telson) con una precisión de $0.05 \mathrm{~mm}$. La LT de los camarones fue categorizada en cinco clases de tamaño.
La búsqueda de ectoparásitos se realizó bajo lupa estereoscópica en la cavidad branquial, filamentos branquiales, paredes externas e internas del cefalotórax, pereiópodos y paredes del abdomen y pleópodos. Para cada hospedero se registró número de ectoparásitos y microhábitat. La magnitud del parasitismo se determinó a través de la prevalencia, intensidad media y ámbito del parasitismo (sensu Margolis et al. 1982). Los parásitos fueron fijados en alcohol al $70 \%$, etiquetado y determinado hasta nivel específico, mediante comparación con material conservado en el Museo de La Plata, Argentina y literatura especializada (Ringuelet 1985). Todo el material biológico se depositó en el Museo de Zoología de la Universidad de Concepción, en la colección general de Anélidos.

La co-presencia de sanguijuelas fue analizada mediante las diferencias entre las frecuencias observadas y esperadas de infecciones dobles, con tablas de contingencia de 2 x 2 y la significancia estadística a través del estadístico Chi-cuadrado (Zar 1984). Las frecuencias esperadas de infecciones dobles, fueron calculadas bajo el procedimiento tradicional que consiste en calcular las infecciones dobles esperadas por azar a partir de la multiplicación de las prevalencias observadas (Lafferty et al. 1994).

La distribución de la prevalencia según la ontogenia del hospedero, se evaluó a través de la comparación de las clases de LT de camarones parasitados y no parasitados y entre especies de ectoparásitos, para lo cual se aplicó la prueba de Kolmogorov-Smirnov para dos muestras (Siegel y Castellan 1988). También se comparó la variación de la intensidad media entre las distintas clases de tamaño, para lo cual se utilizó una prueba de correlación de Spearman (Zar 1984). Un análisis de la varianza de la LT de los camarones parasitados con distintas parasitosis y no parasitados, permitió evaluar si los taxones parasitarios ocupaban hospederos de distinto tamaño (Sokal y Rohlf 1981).

Se indagó si existían diferencias en las medias de la LT de los camarones de distinto sexo, mediante una prueba $t$ de Student. Las variaciones de la distribución de las infecciones 
según el sexo del hospedero, se evaluó mediante análisis de tablas de contingencia de 2 x 2 y la significancia estadística mediante Chi-cuadrado. La variación de la intensidad promedio, según la LT de hospederos de distinto sexo, se determinó a través de pruebas de correlación de Spearman de una cola (Zar 1984).

\section{RESULTADOS}

Se encontró un total de 209 ejemplares de sanguijuelas pertenecientes a dos especies congenéricas de la familia Glossiphoniidae: Helobdella triserialis (Blanchard, 1849) y $H$. duplicata (Moore, 1911). De los 165 camarones examinados el $47 \%$ presentaba ectoparásitos. De estos 77 camarones parasitados, el 85 $\%$ se encontraba infectado por $H$. triserialis, el $10 \%$ por $H$. duplicata y el $5 \%$ por ambas especies. H. triserialis solo se encontró en los pleópodos, con una intensidad media de 2.9 y un ámbito entre 1 a 11 individuos. H. duplicata en cambio, se encontró solo en los filamentos branquiales con una intensidad media de $1.7 \mathrm{y}$ un ámbito entre 1-4 individuos.

La frecuencia observada de camarones con infecciones dobles no fue significativamente distinta de las esperadas por azar $\left(\chi^{2}=0.4, \mathrm{gl}=\right.$ $1, p>0.05)$. Sin embargo, cuando se consideró la sobreposición de microhábitat como copresencia, la frecuencia observada de infecciones multiespecíficas fue significativamente menor a la esperada (Prueba exacta de Fisher, $\mathrm{p}=0.0006$ ). Se consideró la alta prevalencia e intensidad de $H$. triserialis, como alta habilidad competitiva y dominante sobre $H$. duplicata (Lafferty et al. 1994).

La longitud total de los camarones rerecolectados fluctuó entre 50 y $150 \mathrm{~mm}$. Hubo variaciones significativas de la prevalencia total según la LT de los camarones parasitados y no parasitados (Kolmorov-Smirnov, $\mathrm{D}_{\max (77,88)}=$ $0.229, \mathrm{p}<0.05$ (Fig. 1). Sin embargo, la LT promedio de los camarones no parasitados fue mayor a la LT promedio de los parasitados por H. triserialis (Tukey, $\mathrm{F}_{(2,158)}=7.7, \mathrm{p}<0.01$ ). Con respecto a la intensidad media se observó una

\section{A) Total}
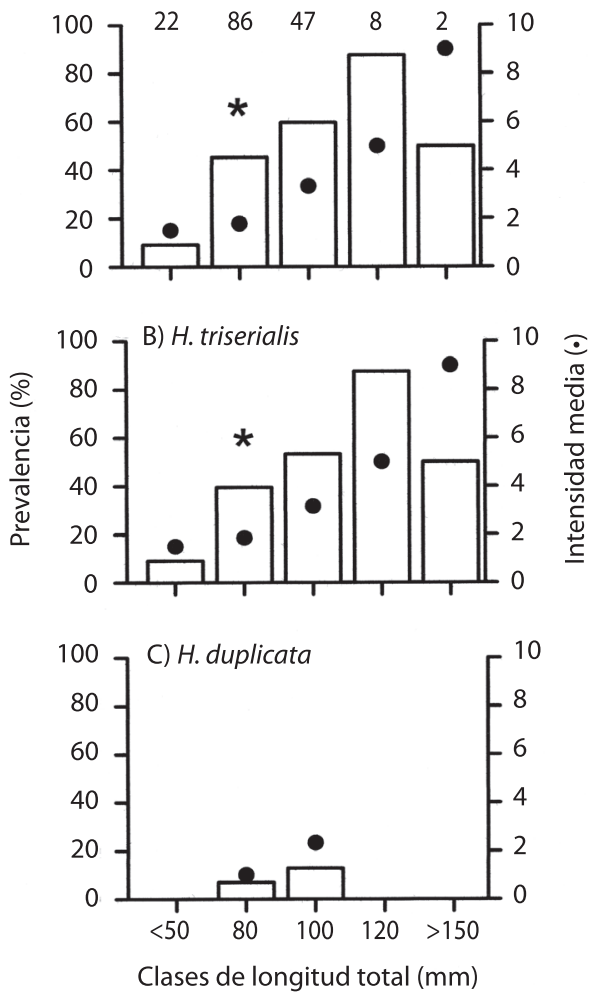

Fig. 1. Prevalencia ( $\%$ de inteccion del hospedero) e intensidad media de infección de (A) Helobdella spp., (B) $H$. triserialis y (C) H. duplicata según el tamaño corporal de su hospedero, C. caementarius. Los números sobre las barras corresponden a la frecuencia absoluta de camarones capturados en cada clase de tamaño corporal y el asterisco indica la clase de longitud total donde se separan dos grupos.

Fig. 1. Prevalence (\% infection from the host sample) and mean infection intensity of (A) Helobdella spp. (B) $H$. triserialis and (C) H. duplicata according to the body size of the host $C$. caementarius. The numbers above the bars correspond to the absolute frequency of the shrimp capturated in each class of total length and the asterisk indicates total length class separating two groups.

tendencia general a aumentar con el tamaño corporal de los hospederos (Correlación de Spearman $\left.r_{s}=1, p=0.04\right)$, tendencia que se mantiene al comparar las especies de sanguijuelas por separado y entre sexos del hospedero (con excepción de la primera clase de longitud total en machos (Fig. 1 y 2). 
Del total de camarones examinados, 82 fueron machos y 83 hembras, con igual distribución de LT promedio ( $\mathrm{t}-$ Student, $\mathrm{t}=1.97$, $\mathrm{gl}=163, \mathrm{p}=0.4)$. Existe una mayor proporción de hembras parasitadas $\left(\chi^{2}=3.8, \mathrm{gl}=1\right.$, $\mathrm{p}=0.05$ ), sin embargo cuando se consideran las infecciones por especie de sanguijuela, solo se mantiene la tendencia para las hembras parasitadas por $H$. triserialis $\left(\chi^{2}=2.8, \mathrm{gl}=1\right.$, $\mathrm{p}<0.05)$. Hubo variación significativa de la prevalencia total según la LT de las hembras parasitadas (Kolmorov-Smirnov, $\mathrm{D}_{\max (45,38)}=0.361$, $\mathrm{p}<0.01$ ), en cambio los machos mostraron una prevalencia total constante e independiente de la LT de los hospederos (Fig. 2).

\section{DISCUSIÓN}

Normalmente las sanguijuelas de agua dulce son depredadores de organismos pequeños y pueden encontrarse como ectoparásitos de aves acuáticas, reptiles, anfibios, anélidos, moluscos y larvas de insectos (Mann 1962, Davies et al. 1997). En el caso de Helobdella, uno de los géneros mas diversificados en Sudamérica (Siddall y Borda 2003), la mayoría de sus especies se alimentan de invertebrados acuáticos y solo unas pocas especies se alimentan de fluidos corporales. Por otra parte, escasos trabajos mencionan a sanguijuelas como ectoparásitos de crustáceos (Kutschera 1988), por lo que este trabajo sería el primer registro de hirudíneos en el decápodo C. caementarius.

La presencia y transmisión de estas sanguijuelas, probablemente se encuentra asociada a las especies de la comunidad donde habitan y en la cual se encuentran moluscos gastrópodos; peces y anfibios (Bahamonde y Vila 1971). En Chile los hirudíneos han sido utilizados como indicadores de calidad de agua siendo característicos de sectores muy intervenidos (Figueroa et al. 2003). Por ello factores abióticos como la irregularidad del caudal, presencia de efluentes, además del régimen exorreico del río Limarí, podrían contribuir a la presencia de Helobdella en $C$. caementarius.

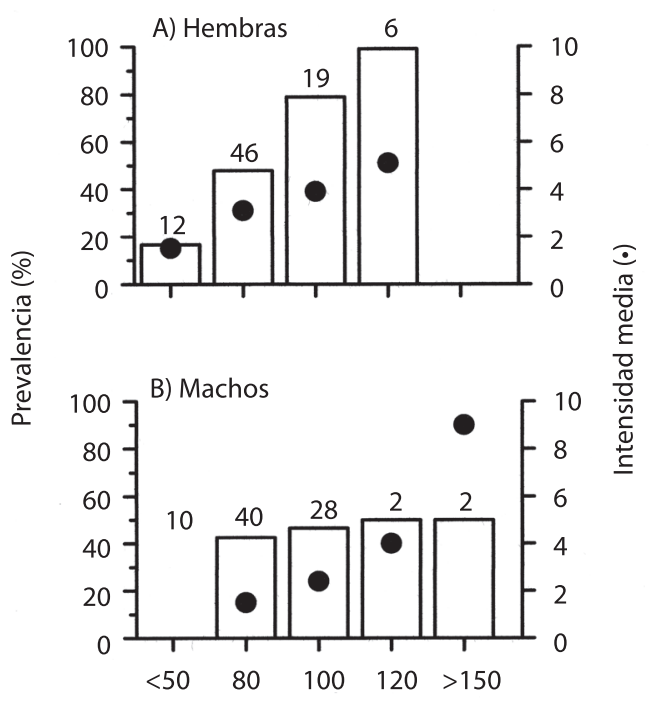

Clases de longitud total ( $\mathrm{mm})$

Fig. 2. Prevalencia (\% de infección) e intensidad media de infección de hirudíneos de ambos sexos, según el tamaño corporal de su hospedero C. caementarius (A) hembras, (B) machos Los números sobre las barras corresponde a la frecuencia absoluta de camarones capturados en cada clase de tamaño corporal.

Fig. 2. Hirudinea prevalence ( $\%$ infection) and mean infection intensity according to the body size of the host $C$. caementarius (A) females (B) males. The numbers above the bars correspond to the absolute frequency of the shrimp captured in each total length class.

Estos hirudíneos muestran una conducta similar a la de isópodos parásitos de crustáceos Palaemonidae descrita por Grassini (1994), quienes utilizan como microhábitats áreas blandas como son la base de pleópodos y branquias. Sin embargo, ambas especies de Helobdella, muestran una distribución espacial diferencial sobre el hospedero, encontrándose en microhábitats distintos en la totalidad de los casos. Esta segregación del hábitat podría ser consecuencia de interacciones antagónicas, lo que estaría sustentado por a lo menos tres observaciones. Primero, las frecuencias observadas de infecciones dobles fueron significativamente menores a las esperadas cuando se considera el microhábitat como lugar de co-presencia. 
Segundo, C. caementarius es susceptible en un ámbito similar de longitud total, de ser parasitado por ambas especies de sanguijuelas, lo que aumentaría aún más la probabilidad de encuentro entre parásitos de distintas especies en un mismo hospedero (Esch y Fernández 1993, Combes 2001). Tercero, es esperable que especies congenéricas utilicen recursos de manera similar, como sucede en las organizaciones tipo gremio (sensu Root 1973), donde es usual encontrar interacciones interespecíficas. Finalmente, los altos valores de prevalencia e intensidad de $H$. triserialis en la base de los pleópodos, sugieren que esta especie podría ser dominante con respecto a $H$. duplicata (Lafferty et al. 1994) y que este microhábitat podría ser más adecuado para el desarrollo de estos hirudíneos.

La longitud total del hospedero resultó ser un buen predictor de las variaciones de la intensidad media y prevalencia del parasitismo por hirudíneos (Fig. 1 y 2). La mayor intensidad encontrada en los camarones de mayor tamaño corporal, puede ser explicada por la mayor disponibilidad espacial asociada a cantidad de alimento y calidad de microhábitat. Por otra parte y ya que los Glossiphonidae manifiestan cuidado parental de sus crías, los estados jóvenes podrían reubicarse dentro del mismo hospedero, aumentando la intensidad sin que se vea afectada la prevalencia. Las variaciones de la prevalencia según la LT del hospedero estaría explicada por el parasitismo de $H$. triserialis en hospederos hembras (Fig. 1 y 2). Es plausible que $H$. triserialis explique la tendencia general de la variación de la prevalencia ya que representa el $85 \%$ de las infecciones. Es necesario entonces analizar una muestra de hospederos de mayor tamaño que permita estimar variaciones en la prevalencia de $H$. duplicata. La variación exclusiva de la prevalencia con la LT de las hembras, podría estar explicada por características morfológicas y conductuales del hospedero. Las hembras de C. caementarius poseen un mayor desarrollo del abdomen y muestran una mayor vagilidad que los machos por las migraciones de desove que realizan a la desembocadura de los ríos (Bahamonde y Vila
1971) aumentando la probabilidad del asentamiento y adquisición de sanguijuelas.

\section{AGRADECIMIENTOS}

Al Museo de La Plata, Argentina por el préstamo del material de $H$. duplicata y $H$. triserialis y al Proyecto DIUC 200.113.055-1.0 de la Dirección de Investigación de la Universidad de Concepción, Chile, por financiamiento.

\section{RESUMEN}

Sanguijuelas de las dos especies Helobdella duplicata y H. triserialis (ambas Glossiphonidae) parasitan al camarón de río Cryphiops caementarius en río Limarí, Chile. El $47 \%$ de los camarones estaba parasitado por una o ambas especies de hirudíneos. H. triserialis fue encontrada solo en los pleópodos con los mayores valores de prevalencia (42\%), intensidad (2.9) y ámbito (1-11). H. duplicata se encontró solamente en los filamentos branquiales con una prevalencia de $7 \%$ intensidad de 1.7 y un ámbito de $1-4$. La longitud total y el sexo del hospedero, explicarían las variaciones de la intensidad y prevalencia de las infecciones. Sin embargo, cuando se considera el sexo del hospedero, la prevalencia solo aumenta con el tamaño de las hembras. Las características morfológicas de las hembras podrían explicar la mayor intensidad de $H$. triserialis en la base de los pleópodos, algo relacionado a su vez con un mayor desarrollo del abdomen.

Palabras clave: Helobdella, sanguijuelas, Palaemonidae, Cryphiops caementarius, ectoparásitos, Chile.

\section{REFERENCIAS}

Amaya, de G. \& A. Guerra. 1976. Especies de camarones de los ríos norteños del Perú y su distribución. In Conv. Minist. Pesq. Univ. Trujillo Perú, Ministerio de Pesquería, Lima, Perú.

Bahamonde, N. \& I. Vila. 1971. Sinopsis sobre la biología del camarón de río del norte. Biol. Pesq. 5: 1-60.

Baudoin, M. 1975. Host castration as a parasitic strategy. Evolution 29: 335-352.

Calvo-Ugartebur, G. \& C.D. McQuaid. 1998. Parasitism and introduced species: epidemiology of trematodes in the intertidal mussels Perna perna and Mytilus galloprovincialis. J. Exp. Mar. Biol. Ecol. 220: 47-65. 
Combes, C. 2001. Parasitism. The Ecology and evolution of intimate interactions. Chicago, Illinois, EEUU. $552 \mathrm{p}$.

Coop, R.L., M.A. Taylor, D.E. Jacobs \& F. Jackson. 2002. Ectoparasites: recent advances in control. Trends Parasitol. 18: 55-56.

Davies, R.W., N.J. McLoughlin \& J.H. Oosthuizen. 1997. The life-cycle and feeding of the African freshwater leech Helobdella conifera (Glossiphoniidae). S. Afr. J. Zool. 32: 1-4.

Esch, G. \& J. Fernández. 1993. A Functional Biology of Parasitism: ecological and evolutionary implications. Chapman \& Hall, Londres, Inglaterra. 337 p.

Figueroa, R., C. Valdovinos, E. Araya \& O. Parra. 2003. Macroinvertebrados bentónicos como indicadores de calidad de agua de ríos del sur de Chile. Rev. Chil. Hist. Nat. 76: 275-285.

Grassini, C. 1994. Estudios preliminares de Telotha henselii (Crustacea: Isopoda: Cymothoidae) parásito de camarones palaemónidos. An. Museo Hist. Nat. Valparaíso 22: 81-89.

Gullo, B.S. 2003. Ciclo reproductor de Helobdella triserialis (Hirudinea, Glossiphoniidae), Provincia de Buenos Aires, Argentina. Rev. Museo de La Plata Zool. 15: 1-7.

Jara, C. 1997. Antecedentes sobre el desarrollo de la carcinología en Chile. Invest. Mar. Valparaíso 25: 245-254.

Kutschera, U. 1988. A new leech species from North America, Helobdella californica nov. sp. (Hirudinea: Glossiphoniidae). Zool. Anz. 220: 173-178.

Lafferty, K.D., D.T. Sammond \& A.M. Kuris. 1994. Analysis of trematode communities. Ecology 75: 2275-2285.

Mann, K.H. 1962. Leeches (Hirudinea). Their structure, physiology, ecology and embriology. Pergamon, Nueva York, EEUU. 201 p.

Margolis, L., G.W. Esch, J.C. Holmes, A. Kuris \& G. Schad. 1982. The use of ecological terms parasitology. J. Parasitol. 68: 131-133.
Meruane, J. 1994. Los Primeros Pasos del Cultivo Comercial de Camarón de Río. Aquanoticias Internac. (JulioSeptiembre): 32-33.

Muñoz, G. \& M. George-Nascimento. 1999. Efectos recíprocos entre napes e isópodos bopíridos en Lenga, Chile. Rev. Chil. Hist. Nat. 72: 49-56.

Olmos, V. \& M. George-Nascimento. 1997. El gremio de larvas de Digenea en el caracol del sur de Chile Chilina dombeyana: ¿qué indica la tasa metabólica de los hospedadores parasitados? Rev. Chil. Hist. Nat. 70: 109-118.

Retamal, M.A. 1981. Catálogo Ilustrado de Crustáceos Decápodos de Chile. Gayana Zool. 44: 1-110.

Ringuelet, R. 1985. Sinopsis de los Hirudineos de Chile (ANELIDA). Bol. Soc. Biol. Concepción 56: 163 179.

Root, R.B. 1973. Organization of a plant-arthropod association in simple and diverse habitats: the fauna of collards (Brassica oleracea). Ecol. Monogr. 43: 95- 124

Sasal, P., P. Durand, E. Faliex \& S. Morand. 2000. Experimental approach to the importance of parasitism in biological conservation. Mar. Ecol-Prog. Ser. 198: 293-302.

Sawyer, R.T. 1986. Leech Biology and Behaviour. Clarendon, Oxford, Inglaterra. 1065 p.

Siddal, M.E. \& E. Borda. 2003. Phylogeny and revision of the leech Helobdella. (Glossiphonidae) based on mitochondrial gene sequences and morphological data and a special consideration of the triserialis complex. Zool. Scr. 32: 23-33.

Siegel, S. \& N.J. Castellan. 1988. Nonparametric statistics for the behavioral sciences. McGraw-Hill, Nueva York, EEUU. 399 p.

Sokal, R.R. \& F.J. Rohlf. 1981. Biometry: The principles and practice of statistics in biological research. Freeman W.H., Nueva York, EEUU. 959 p.

Zar, J.H. 1984. Biostatistical analysis. Prentice-Hall, New Jersey, USA. 718 p. 